PROCEEDINGS OF THE

AMERICAN MATHEMATICAL SOCIETY

Volume 131, Number 3, Pages 801-813

S 0002-9939(02)06574-7

Article electronically published on July 26, 2002

\title{
CANONICAL FACTORIZATION OF CONTINUOUS FUNCTIONS ON THE $d$-TORUS
}

\author{
TORSTEN EHRHARDT AND CORNELIS V. M. VAN DER MEE
}

(Communicated by Joseph A. Ball)

\begin{abstract}
In this article we study the canonical factorization of continuous complex-valued functions on the $d$-dimensional torus belonging to a weighted Wiener algebra with respect to a linear order on the $d$-tuples of integers. It is proved that a function has a canonical factorization in this algebra if and only if it has a logarithm belonging to this algebra. A second characterization is given in terms of winding numbers. Moreover, the maximal ideal spaces of the relevant Banach algebras are identified.
\end{abstract}

\section{INTRODUCTION}

Let $\mathbb{T}$ denote the unit disk in the complex plane $\mathbb{C}, \mathbb{T}^{d}$ the $d$-dimensional torus, $\mathbb{Z}$ the set of all integers and $\mathbb{Z}^{d}$ the $d$-dimensional integer lattice. Consider a weight $\boldsymbol{\beta}=\left\{\beta_{i}\right\}_{i \in \mathbb{Z}^{d}}$ satisfying the condition $1 \leq \beta_{i+j} \leq \beta_{i} \beta_{j}$ for all $i, j \in \mathbb{Z}^{d}$. A complexvalued function $a$ on $\mathbb{T}^{d}$ is said to be in the $\boldsymbol{\beta}$-weighted Wiener class $\mathbb{W}_{\boldsymbol{\beta}}^{d}$ if it can be represented in the form

$$
a(z)=\sum_{i=\left(i_{1}, \ldots, i_{d}\right) \in \mathbb{Z}^{d}} a_{i} z_{1}^{i_{1}} \cdots z_{d}^{i_{d}}, \quad z=\left(z_{1}, \ldots, z_{d}\right) \in \mathbb{T}^{d},
$$

where

$$
\|a\|_{\mathbb{W}_{\beta}^{d}}:=\sum_{i \in \mathbb{Z}^{d}} \beta_{i}\left|a_{i}\right|<\infty .
$$

Then $\mathbb{W}_{\boldsymbol{\beta}}^{d}$ is a commutative Banach algebra. If $\beta_{i} \equiv 1$, then we will write $\mathbb{W}^{d}$ for brevity.

Let $\succeq$ be a linear order on $\mathbb{Z}^{d}$ such that $\mathbb{Z}^{d}$ becomes an ordered group [18] (i.e., $i+l \succeq j+l$ whenever $i \succeq j$ and $i, j, l \in \mathbb{Z}^{d}$ ). By a canonical factorization of $a$ in $\mathbb{W}_{\boldsymbol{\beta}}^{d}$ (with respect to $\succeq$ ) we mean a factorization of $a \in \mathbb{W}_{\boldsymbol{\beta}}^{d}$ of the type

$$
a(z)=a_{-}(z) a_{+}(z), \quad z \in \mathbb{T}^{d},
$$

where $a_{ \pm}$and $1 / a_{ \pm}$belong to the closed subalgebra

$$
\mathbb{W}_{\boldsymbol{\beta}}^{d, \pm}=\left\{c \in \mathbb{W}_{\boldsymbol{\beta}}^{d}: c_{i}=0 \text { for all }( \pm i) \prec 0\right\}
$$

Received by the editors July 18, 2001 and, in revised form, October 12, 2001.

1991 Mathematics Subject Classification. Primary 46J10; Secondary 43A20.

Key words and phrases. Canonical factorization, Wiener algebra, maximal ideal space.

This research was partially supported by INdAM-GNCS and MURST. 
Remark that the factors in a canonical factorization are uniquely determined up to a nonzero multiplicative constant.

The maximal ideal space of $\mathbb{W}_{\boldsymbol{\beta}}^{d} \subseteq \mathbb{W}^{d}$ can be shown to be homeomorphic to the non-empty, compact set

$$
\Omega_{\boldsymbol{\beta}}=\left\{z=\left(z_{1}, \ldots, z_{d}\right) \in \mathbb{C}^{d}: \sup _{i=\left(i_{1}, \ldots, i_{d}\right) \in \mathbb{Z}^{d}} \frac{\left|z_{1}\right|^{i_{1}} \cdots\left|z_{d}\right|^{i_{d}}}{\beta_{i}}<\infty\right\} .
$$

In fact, the Gelfand transform of $a \in \mathbb{W}_{\boldsymbol{\beta}}^{d}$ is given by the continuous function

$$
\hat{a}(z)=\sum_{i=\left(i_{1}, \ldots, i_{d}\right) \in \mathbb{Z}^{d}} a_{i} z_{1}^{i_{1}} \cdots z_{d}^{i_{d}}, \quad z=\left(z_{1}, \ldots, z_{d}\right) \in \Omega_{\boldsymbol{\beta}} .
$$

Remark that $\hat{a}(z)=a(z)$ for $z \in \mathbb{T}^{d}$. The maximal ideal space of $\mathbb{W}^{d}$ is equal to the torus $\mathbb{T}^{d}$.

Canonical factorizations have been studied for $d=1$ since the seminal paper of Krein [14. The necessary and sufficient condition for $a$ to have a canonical factorization in $\mathbb{W}_{\boldsymbol{\beta}}^{1}$ (derived in [14] for $\beta_{i} \equiv 1$ ) is that $\hat{a}(z) \neq 0$ for $z \in \Omega_{\boldsymbol{\beta}}$ and the contour $\{a(z): z \in \mathbb{T}\}$ has winding number zero with respect to the origin. An equivalent way of expressing this necessary and sufficient condition is to state that $a=\exp (b):=\sum_{n=0}^{\infty}\left(b^{n} / n\right.$ !) for some $b \in \mathbb{W}_{\boldsymbol{\beta}}^{1}$. For $d=1$, the maximal ideal space of $\mathbb{W}_{\boldsymbol{\beta}}^{1}$ coincides with the annulus $\left\{z \in \mathbb{T}: r_{-} \leq|z| \leq r_{+}\right\}$, where $\left(r_{ \pm}\right)^{ \pm 1}$ is the limit of $\beta_{ \pm i}^{1 / i}$ as $i \rightarrow \infty$ 6, 7]. Factorizations of positive definite functions in $\mathbb{W}^{2}$ have been studied in [9]. In [19, the closely related problem of giving conditions under which a Toeplitz operator defined on $\ell^{p}(M)$, where $M$ is a cone in $\mathbb{Z}^{d}$, is a Fredholm operator, has been studied without taking into account weights. Some partial results of this type can also be found in 2. In this article we generalize the conditions for the canonical factorization in Banach algebras of functions on the circle to multi-index weighted Wiener algebras, i.e., to the case $d \geq 2$ and $\beta_{i} \not \equiv 1$.

\section{The MAIN RESUlT AND COROLlaries}

Let $a$ be a continuous complex-valued function on the torus $\mathbb{T}^{d}$ such that $a(t) \neq 0$ for all $t \in \mathbb{T}^{d}$. For $s=1, \ldots, d$, the winding number $\operatorname{wind}_{s}(a)$ is defined as the winding number of the contour $\left\{a\left(z_{1}, \ldots, z_{s-1}, t, z_{s+1}, \ldots, z_{d}\right): t \in \mathbb{T}\right\}$ with respect to the origin. Note that this definition does not depend on the particular choice of $\left(z_{1}, \ldots, z_{s-1}, z_{s+1}, \ldots, z_{d}\right) \in \mathbb{T}^{d-1}$.

The main result of this article is the following theorem.

Theorem 2.1. Let $\boldsymbol{\beta}=\left\{\beta_{i}\right\}_{i \in \mathbb{Z}^{d}}$ be a weight satisfying $1 \leq \beta_{i+j} \leq \beta_{i} \beta_{j}\left(i, j \in \mathbb{Z}^{d}\right)$ and $\succeq$ a linear order on $\mathbb{Z}^{d}$ such that $\mathbb{Z}^{d}$ becomes an ordered group. Suppose $a \in \mathbb{W}_{\boldsymbol{\beta}}^{d}$. Then the following three statements are equivalent:

(a) a has a canonical factorization in $\mathbb{W}_{\boldsymbol{\beta}}^{d}$ with respect to $\succeq$;

(b) a can be written in the form $a=\exp (b)$ for some $b \in \mathbb{W}_{\boldsymbol{\beta}}^{d}$;

(c) $\hat{a}(z) \neq 0$ for all $z \in \Omega_{\boldsymbol{\beta}}$ and $\operatorname{wind}_{s}(a)=0$ for all $s=1, \ldots, d$.

Statements (b) and (c) do not depend on the underlying linear order. Hence the existence of the canonical factorization in $\mathbb{W}_{\boldsymbol{\beta}}^{d}$ is independent of the linear order. From the proof of the implication $(b) \Rightarrow$ (a) given below it will become clear that the factors $a_{ \pm}$, which depend on the linear order, can be defined explicitly. 
A sufficient, but not necessary, condition for statement (b) is that $\log (z)$ can be defined continuously on the set $\left\{\hat{a}(z): z \in \Omega_{\boldsymbol{\beta}}\right\}$.

Let us continue with the proof of the trivial implications (b) $\Rightarrow(\mathrm{a})$ and (b) $\Rightarrow(\mathrm{c})$.

Proof of the implication (b) $\Rightarrow(\mathrm{a})$. Assume $a=\exp (b)$ for some $b \in \mathbb{W}_{\boldsymbol{\beta}}^{d}$, and let $b(z)=\sum_{i \in \mathbb{Z}^{d}} b_{i} z^{i}$ for $z \in \mathbb{T}^{d}$. Then

$$
a(z)=\exp \left(b_{-}(z)\right) \exp \left(b_{+}(z)\right), \quad z \in \mathbb{T}^{d},
$$

where $b_{ \pm}(z)=\sum_{i \in \mathbb{Z}^{d}} c_{i}^{( \pm)} z^{i}$ for $z \in \mathbb{T}^{d}$ with $c_{0}^{( \pm)}=\frac{1}{2} b_{0}, c_{i}^{( \pm)}=b_{i}$ if $( \pm i) \succ 0$ and $c_{i}^{( \pm)}=0$ if $( \pm i) \prec 0$, is a canonical factorization of $a$ in $\mathcal{W}_{\boldsymbol{\beta}}^{d}$.

Proof of the implication $(\mathrm{b}) \Rightarrow(\mathrm{c})$. Assume that $a=\exp (b)$ for $b \in \mathbb{W}_{\boldsymbol{\beta}}^{d}$. Then $a(z) \neq 0$ for $z \in \Omega_{\boldsymbol{\beta}}$. For $s=1, \ldots, d$ and any fixed $z_{1}, \ldots, z_{s-1}, z_{s+1}, \ldots, z_{d} \in \mathbb{T}$, the function $a_{s}(t):=a\left(z_{1}, \ldots, z_{s-1}, t, z_{s+1}, \ldots, z_{d}\right), t \in \mathbb{T}$, is the exponential of the function $b_{s}(t):=b\left(z_{1}, \ldots, z_{s-1}, t, z_{s+1}, \ldots, z_{d}\right), t \in \mathbb{T}$. Hence wind ${ }_{s}(a)=0$.

The proof of the implication $(c) \Rightarrow(b)$ will be given Section 3. For the implication $(\mathrm{a}) \Rightarrow(\mathrm{b})$ we will give two proofs, one in Section 4 and another in Section 5

In the following corollary, we answer the question whether (under the obviously necessary condition) the existence of a factorization in $\mathbb{W}^{d}$ implies the existence of a factorization in $\mathbb{W}_{\boldsymbol{\beta}}^{d}$. It was already established in [15] (and in [8] for more specific weights) under the additional assumption that $\log (z)$ can be defined continuously on the set $\left\{\hat{a}(z): z \in \Omega_{\boldsymbol{\beta}}\right\}$.

Corollary 2.2. Let $\boldsymbol{\beta}=\left\{\beta_{i}\right\}_{i \in \mathbb{Z}^{d}}$ be a weight satisfying $1 \leq \beta_{i+j} \leq \beta_{i} \beta_{j}\left(i, j \in \mathbb{Z}^{d}\right)$ and $\succeq$ a linear order of $\mathbb{Z}^{d}$ such that $\mathbb{Z}^{d}$ becomes an ordered group. Suppose $a \in \mathbb{W}_{\boldsymbol{\beta}}^{d}$ and $\hat{a}(z) \neq 0$ for all $z \in \Omega_{\boldsymbol{\beta}}$. Then if a has a canonical factorization in $\mathbb{W}^{d}$, the factors $a_{ \pm}$and $1 / a_{ \pm}$belong to $\mathbb{W}_{\boldsymbol{\beta}}^{d, \pm}$.

For the proof it suffices to note that due to the assumption $\hat{a}(z) \neq 0$ for all $z \in \Omega$, condition (c) in Theorem 2.1 reduces to the winding number condition, which does not depend on the underlying weight.

Another corollary, which appears for $\beta_{i} \equiv 1$ in [19], is the following. It deals with the existence of factorizations which are not necessarily canonical.

Corollary 2.3. Let $\boldsymbol{\beta}=\left\{\beta_{i}\right\}_{i \in \mathbb{Z}^{d}}$ be a weight satisfying $1 \leq \beta_{i+j} \leq \beta_{i} \beta_{j}\left(i, j \in \mathbb{Z}^{d}\right)$ and $\succeq$ a linear order of $\mathbb{Z}^{d}$ such that $\mathbb{Z}^{d}$ becomes an ordered group. Suppose $a \in \mathbb{W}_{\boldsymbol{\beta}}^{d}$ and $\hat{a}(z) \neq 0$ for $z \in \Omega_{\boldsymbol{\beta}}$. Then a allows the representation

$$
a(z)=a_{-}(z) z^{\kappa} a_{+}(z), \quad z \in \mathbb{T}^{d},
$$

where $a_{ \pm}$and $1 / a_{ \pm}$belong to $\mathbb{W}_{\beta}^{d, \pm}, z^{\kappa}=z_{1}^{\kappa_{1}} \cdots z_{d}^{\kappa_{d}}$, and $\kappa_{s}=\operatorname{wind}_{s}(a)$.

In order to prove Corollary 2.3 it suffices to apply the implication (c) $\Rightarrow$ (a) of Theorem 2.1 to the function $b$ defined by $b(z)=z^{-\kappa} a(z), z \in \mathbb{T}^{d}$.

In what follows, it will be more convenient not to work with the Banach algebras $\mathbb{W}_{\boldsymbol{\beta}}^{d}$ and $\mathbb{W}_{\boldsymbol{\beta}}^{d, \pm}$ of functions, but with Banach algebras consisting of the corresponding sequences of Fourier coefficients. We also prefer to give the following definitions in the somewhat wider framework of arbitrary additive groups.

Let $N$ be an additive group. An admissible weight $\boldsymbol{\beta}$ on $N$ is a sequence $\boldsymbol{\beta}=$ $\left\{\beta_{i}\right\}_{i \in N}$ of positive real numbers for which $1 \leq \beta_{i+j} \leq \beta_{i} \beta_{j}$ for all $i, j \in N$. Let 
$\mathcal{W}_{\boldsymbol{\beta}}^{N}$ denote the Banach algebra of all sequences $\boldsymbol{a}=\left\{a_{i}\right\}_{i \in N}$ of complex numbers for which at most countably many terms are nonzero and

$$
\|\boldsymbol{a}\|_{\mathcal{W}_{\boldsymbol{\beta}}^{N}}:=\sum_{i \in N} \beta_{i}\left|a_{i}\right|<\infty
$$

where the multiplication is defined as the convolution of two sequences.

If, in addition, $N$ is an ordered group with respect to $\succeq$, then denote by

$$
N_{ \pm}=\{i \in N:( \pm i) \succeq 0\}
$$

the semi-groups of all non-negative (resp. non-positive) elements. The Banach algebras $\mathcal{W}_{\boldsymbol{\beta}}^{N_{ \pm}}$are the sets of all sequences $\boldsymbol{a}=\left\{a_{i}\right\}_{i \in N_{ \pm}}$for which

$$
\|\boldsymbol{a}\|_{\mathcal{W}_{\boldsymbol{\beta}}^{N_{ \pm}}}:=\sum_{i \in N_{ \pm}} \beta_{i}\left|a_{i}\right|<\infty
$$

where the multiplication is again defined as convolution.

\section{The MaXimal IDEAL SPACE OF $\mathcal{W}_{\boldsymbol{\beta}}^{N}$ FOR $N=\mathbb{Z}^{d}$}

We start with some convex analysis (see, e.g., [12]). A subadditive function on an additive group $N$ is a function $f: N \rightarrow \mathbb{R}$ such that $f(x+y) \leq f(x)+f(y)$ for all $x, y \in N$. A sublinear function on $\mathbb{R}^{d}$ is a function $F: \mathbb{R}^{d} \rightarrow \mathbb{R} \cup\{+\infty\}$ which satisfies the following conditions:

(i) $F(0)=0$;

(ii) $F(x+y) \leq F(x)+F(y)$ for all $x, y \in \mathbb{R}^{d}$;

(iii) $F(\lambda x)=\lambda F(x)$ for all $x \in \mathbb{R}^{d}$ and $\lambda>0$.

A sublinear function $F$ taking values only in $\mathbb{R}$ will be called finite. Finite sublinear functions are convex and hence continuous.

Proposition 3.1. Let $f: \mathbb{Z}^{d} \rightarrow \mathbb{R}$ be a subadditive function. Then there exists a (unique) finite sublinear function $F: \mathbb{R}^{d} \rightarrow \mathbb{R}$ such that

$$
F(i)=\lim _{n \rightarrow \infty} \frac{f(n i)}{n} \quad \text { for all } i \in \mathbb{Z}^{d} .
$$

Proof. For fixed $i \in \mathbb{Z}^{d}$, the function $f_{i}: \mathbb{Z} \rightarrow \mathbb{R}, n \mapsto f(n i)$, is subadditive on $\mathbb{Z}$. From [11, Chap. V] it follows that the limit (3.1) exists. Hence $F$ is (uniquely) defined on $\mathbb{Z}^{d}$. Note that $F(0)=0$, and $F(n i)=n F(i)$ for all $i \in \mathbb{Z}^{d}$ and $n \in \mathbb{N}$.

Next we extend $F$ to $\mathbb{Q}^{d}$ as follows. For each $x \in \mathbb{Q}^{d}$ there exist $q \in \mathbb{Q}_{+}$and $i \in \mathbb{Z}^{d}$ such that $x=q i$. We define $F(x)=q F(i)$. This definition is correct since it does not depend on the particular choice of $q$ and $i$. Indeed, assume $i_{1}, i_{2} \in \mathbb{Z}^{d}$ and $q_{1}, q_{2} \in \mathbb{Q}_{+}$such that $i_{1} q_{1}=i_{2} q_{2}$. Write $q_{1} / q_{2}=n_{1} / n_{2}$ with $n_{1}, n_{2} \in \mathbb{N}$. Then $n_{1} F\left(i_{1}\right)=F\left(n_{1} i_{1}\right)=F\left(n_{2} i_{2}\right)=n_{2} F\left(i_{2}\right)$ and hence $q_{1} F\left(i_{1}\right)=q_{2} F\left(i_{2}\right)$. Thus there exists a unique function $F: \mathbb{Q}^{d} \rightarrow \mathbb{R}$ satisfying (3.1) and the condition that $F(q i)=q F(i)$ for all $q \in \mathbb{Q}_{+}$and $i \in \mathbb{Z}^{d}$.

We claim that $F$ is subadditive on $\mathbb{Q}^{d}$. Indeed, let $x, y \in \mathbb{Q}^{d}$ and choose $q \in \mathbb{Q}_{+}$ such that both $i=q^{-1} x$ and $j=q^{-1} y$ belong to $\mathbb{Z}^{d}$. Then $f(n i+n j) \leq f(n i)+f(n j)$ implies $F(i+j) \leq F(i)+F(j)$. Thus $F(x+y) \leq F(x)+F(y)$. 
Let $e_{1}, \ldots, e_{d}$ be the canonical unit vectors in $\mathbb{R}^{d}$ and put $e_{-s}=-e_{s}$. Write $x \in \mathbb{Q}^{d}$ as $x=\sum_{s=1}^{d}\left|x_{s}\right| e_{\operatorname{sign}\left(x_{s}\right) s}$. The subadditivity and homogeneity of $F$ imply

$$
F(x) \leq \sum_{s=1}^{d}\left|x_{s}\right| F\left(e_{\operatorname{sign}(s) s}\right) \leq C_{F}\|x\|,
$$

where $C_{F}>0$ is a constant depending only on $F$ but not on $x$. Let $x, y \in \mathbb{Q}^{d}$. Then, again by subadditivity, we obtain $-F(y-x) \leq F(x)-F(y) \leq F(x-y)$. Thus $|F(x)-F(y)| \leq C_{F}\|x-y\|$. Hence $F$ can be (uniquely) continued by continuity to a function on all of $\mathbb{R}^{d}$ which is finite sublinear.

A convex body is a non-empty, compact and convex subset of $\mathbb{R}^{d}$. There exists a one-to-one correspondence between convex bodies of $\mathbb{R}^{d}$ and finite sublinear functions on $\mathbb{R}^{d}$. In fact, given a convex body $K$, the finite sublinear function of support $K$ is defined by

$$
F(x)=\max _{y \in K}\langle x, y\rangle .
$$

Conversely, given a finite sublinear function $F$ on $\mathbb{R}^{d}$, the corresponding convex body can be recovered by

$$
K=\left\{y \in \mathbb{R}^{d}:\langle x, y\rangle \leq F(x) \text { for all } x \in \mathbb{R}^{d}\right\} .
$$

Proposition 3.2. Let $\boldsymbol{\beta}=\left\{\beta_{i}\right\}_{i \in \mathbb{Z}^{d}}$ be an admissible weight. Then

$$
K_{\boldsymbol{\beta}}=\left\{y \in \mathbb{R}^{d}: \sup _{i \in \mathbb{Z}^{d}}\left(\langle i, y\rangle-\log \left(\beta_{i}\right)\right)<\infty\right\}
$$

is a convex body with a supporting function $F$ for which $F(i)=\lim _{n \rightarrow \infty} \frac{\log \left(\beta_{n i}\right)}{n}, i \in \mathbb{Z}^{d}$.

Proof. The function $f(i)=\log \left(\beta_{i}\right)$ is subadditive. By Proposition 3.1 there exists a unique finite sublinear function $F$ for which the above limit relation holds. It suffices to show that $K_{\boldsymbol{\beta}}$ coincides with $K$ defined in (3.4).

Since $\log \left(\beta_{n i}\right) / n \leq \log \left(\beta_{i}\right)$ by subadditivity, it follows that $F(i) \leq \log \left(\beta_{i}\right)$. Hence $K \subseteq K_{\boldsymbol{\beta}}$. Conversely, if $y \in K_{\boldsymbol{\beta}}$, then $\langle i, y\rangle \leq \log \left(\beta_{i}\right)+M$ for all $i \in \mathbb{Z}^{d}$ for a certain constant $M$. Now replace $i$ by $n i$, divide by $n$ and pass to the limit as $n \rightarrow \infty$. It follows that $\langle i, y\rangle \leq F(i)$ for all $i \in \mathbb{Z}^{d}$. Since $F$ is finite sublinear, we obtain $\langle x, y\rangle \leq F(x)$ for all $x \in \mathbb{R}^{d}$. Thus $y \in K$.

We proceed with a description of $\Omega_{\boldsymbol{\beta}}$, which was defined in (1.5), in terms of $K_{\boldsymbol{\beta}}$.

Corollary 3.3. Let $\boldsymbol{\beta}=\left\{\beta_{i}\right\}_{i \in \mathbb{Z}^{d}}$ be an admissible weight. Then $\Omega_{\boldsymbol{\beta}}$ is homeomorphic to $\mathbb{T}^{d} \times K_{\boldsymbol{\beta}}$ :

$$
\Omega_{\boldsymbol{\beta}}=\left\{\left(t_{1} e^{x_{1}}, \ldots, t_{d} e^{x_{d}}\right): t_{1}, \ldots, t_{d} \in \mathbb{T},\left(x_{1}, \ldots, x_{d}\right) \in K_{\boldsymbol{\beta}}\right\} .
$$

In particular, $\Omega_{\boldsymbol{\beta}}$ is a non-empty, compact subset of $(\mathbb{C} \backslash\{0\})^{d}$.

The identification of $\Omega_{\boldsymbol{\beta}}$ with the maximal ideal space of $\mathcal{W}_{\boldsymbol{\beta}}^{N}, N=\mathbb{Z}^{d}$, is provided by the following theorem. For $i \in N$ define the element $\boldsymbol{e}_{i}=\left\{\delta_{i j}\right\}_{j \in N} \in$ $\mathcal{W}_{\boldsymbol{\beta}}^{N}$ where $\delta_{i j}$ is Kronecker's symbol. 
Theorem 3.4. The maximal ideal space of $\mathcal{W}_{\boldsymbol{\beta}}^{N}, N=\mathbb{Z}^{d}$, is homeomorphic to $\Omega_{\boldsymbol{\beta}}$ endowed with the topology induced by $\mathbb{C}^{d}$. In fact, given $z \in \Omega_{\boldsymbol{\beta}}$, the corresponding multiplicative linear functional $T_{z}$ is given by

$$
T_{z}: \boldsymbol{a}=\left\{a_{i}\right\}_{i \in N} \mapsto \sum_{i \in N} a_{i} z^{i}, \quad z^{i}=z_{1}^{i_{1}} \cdots z_{d}^{i_{d}} .
$$

Proof. Since the linear span of $\left\{\boldsymbol{e}_{i}\right\}_{i \in N}$ is dense in $\mathcal{W}_{\boldsymbol{\beta}}^{N}$, there exists a one-toone correspondence between maximal ideals of $\mathcal{W}_{\boldsymbol{\beta}}^{N}$ and sequences $\boldsymbol{h}=\left\{h_{i}\right\}_{i \in N}$ satisfying $h_{0}=1, h_{i+j}=h_{i} h_{j}$ and $\sup _{i \in N}\left|h_{i}\right| / \beta_{i}<\infty$. The multiplicative linear functional corresponding to $\boldsymbol{h}$ is given by $\boldsymbol{a} \mapsto \sum_{i \in N} a_{i} h_{i}$. Note that $h_{i} \neq 0$ for all $i \in N$. Write $i \in \mathbb{Z}^{d}$ as $i=\sum_{s=1}^{d} i_{s} e_{s}$, where $e_{1}, \ldots, e_{d}$ are the canonical unit vectors. It follows that $h_{i}=z_{1}^{i_{1}} \cdots z_{d}^{i_{d}}$ where $z_{s}=h_{e_{s}}$. Hence we proved the one-to-one correspondence between the maximal ideal space of $\mathcal{W}_{\boldsymbol{\beta}}^{N}$ and $\Omega_{\boldsymbol{\beta}}$ as sets.

In order to prove that under this identification the topology of $\Omega_{\beta}$ induced by $\mathbb{C}^{d}$ coincides with the natural topology of the maximal ideal space, we resort to the following result (see, e.g., Sect. 11, Thm. 3 in [16]): Let $\mathcal{M}$ be the maximal ideal space of a Banach algebra topologized in such a way that $\mathcal{M}$ is compact and the function $m \in \mathcal{M} \mapsto m(\boldsymbol{a}) \in \mathbb{C}$ is continuous for all elements $\boldsymbol{a}$ of the Banach algebra. Then this topology coincides with the natural topology of the maximal ideal space. In our situation, the compactness of $\mathcal{M}=\Omega_{\boldsymbol{\beta}}$ follows from Corollary 3.3 Since $\sup _{z \in \Omega_{\boldsymbol{\beta}}}\left|T_{z}(\boldsymbol{a})\right| \leq\|\boldsymbol{a}\|_{\mathcal{W}_{\boldsymbol{\beta}}^{N}}$ and since the linear span of $\left\{\boldsymbol{e}_{i}\right\}_{i \in N}$ is dense in $\mathcal{W}_{\boldsymbol{\beta}}^{N}$, it suffices to prove only the continuity of the maps $z \in \Omega_{\boldsymbol{\beta}} \mapsto T_{z}\left(\boldsymbol{e}_{i}\right)$ for each $i \in N$. Indeed, the functions $z \in \Omega_{\boldsymbol{\beta}} \mapsto T_{z}\left(\boldsymbol{e}_{i}\right)=z_{1}^{i_{1}} \cdots z_{d}^{i_{d}}$ are continuous because $\Omega_{\boldsymbol{\beta}}$ is a subset of $(\mathbb{C} \backslash\{0\})^{d}$.

For a Banach algebra $\mathcal{A}$ with unit element, let $\mathcal{G}(\mathcal{A})$ denote the group of all invertible elements in $\mathcal{A}$, and let $\mathcal{G}_{0}(\mathcal{A})$ denote the connected component of $\mathcal{G}(\mathcal{A})$ containing the unit element. The group $\mathcal{G}_{0}(\mathcal{A})$ is an open and closed normal subgroup of $\mathcal{G}(\mathcal{A})$. The quotient group $\Lambda(\mathcal{A})=\mathcal{G}(\mathcal{A}) / \mathcal{G}_{0}(\mathcal{A})$ is called the abstract index group of the Banach algebra $\mathcal{A}$. If $\mathcal{A}$ is a commutative Banach algebra, then $\mathcal{G}_{0}(\mathcal{A})=\{a=\exp (b): b \in \mathcal{A}\}$ (see [4. Sect. 2.9-15]).

We need the following formulation of the Arens-Royden Theorem. Let $\mathcal{M}$ stand for the maximal ideal space of $\mathcal{A}$ and $\pi^{1}(\mathcal{M})$ for the first cohomotopy group of $\mathcal{M}$, i.e., the group of homotopy classes of continuous functions from $\mathcal{M}$ to the unit circle $\mathbb{T}$ with pointwise multiplication.

Theorem 3.5 (Arens-Royden). Let $\mathcal{A}$ be a commutative Banach algebra with unit element. Then the mapping

$$
\boldsymbol{a} \in \mathcal{G}(\mathcal{A}) \mapsto f(m)=m(\boldsymbol{a}) /|m(\boldsymbol{a})|, m \in \mathcal{M},
$$

induces a group-isomorphism from the abstract index group of $\mathcal{A}$ onto $\pi^{1}(\mathcal{M})$.

Proof. According to the Arens-Royden Theorem [20] (see also [1, 17]) the Gelfand transform induces an isomorphism from the abstract index group of $\mathcal{A}$ onto the abstract index group of the Banach algebra $\mathrm{C}(\mathcal{M})$ of all continuous complex valued functions on $\mathcal{M}$. On the other hand (see, e.g., [4, Thm. 2.18]), the mapping $f(m) \mapsto$ $f(m) /|f(m)|$ induces an isomorphism from $\Lambda(\mathrm{C}(\mathcal{M}))$ onto $\pi^{1}(\mathcal{M})$.

Proof of the implication (c) $\Rightarrow$ (b) of Theorem 2.1. We rely on the natural identification of $\mathcal{W}_{\boldsymbol{\beta}}^{N}, N=\mathbb{Z}^{d}$, with $\mathbb{W}_{\boldsymbol{\beta}}^{d}$. Under this identification, the maximal ideal 
space of $\mathbb{W}_{\boldsymbol{\beta}}^{d}$ is homeomorphic to $\Omega_{\boldsymbol{\beta}}$ and the Gelfand transform of $a \in \mathbb{W}_{\boldsymbol{\beta}}^{d}$ is given by the function $\hat{a}$ defined in (1.6). From the first condition in (c) it follows that $a$ belongs to $\mathcal{G}\left(\mathbb{W}_{\boldsymbol{\beta}}^{d}\right)$. We have to show that the winding number condition implies that $a \in \mathcal{G}_{0}\left(\mathbb{W}_{\boldsymbol{\beta}}^{d}\right)$.

Because $\beta_{i} \geq 1, K_{\boldsymbol{\beta}}$ is a convex body which contains the origin. Hence $K_{\boldsymbol{\beta}}$ can be contracted to the origin. Due to the description of $\Omega_{\beta}$ given in Corollary 3.3 the mapping $[f]_{\sim} \in \pi^{1}\left(\Omega_{\boldsymbol{\beta}}\right) \mapsto\left[\left.f\right|_{\mathbb{T}^{d}}\right]_{\sim} \in \pi^{1}\left(\mathbb{T}^{d}\right)$ is a group-isomorphism. Here $[*]_{\sim}$ stands for the homotopy classes, and $\left.f\right|_{\mathbb{T}^{d}}$ is the restriction of $f$ to $\mathbb{T}^{d}$. Moreover, a group-isomorphism from $\pi^{1}\left(\mathbb{T}^{d}\right)$ onto $\mathbb{Z}^{d}$ is given in terms of winding numbers 13 . In connection with Theorem 3.5 we obtain that the mapping

$$
a \in \mathcal{G}\left(\mathbb{W}_{\boldsymbol{\beta}}^{d}\right) \mapsto\left(\operatorname{wind}_{s}(a)\right)_{s=1}^{d} \in \mathbb{Z}^{d}
$$

induces a group-isomorphism between $\Lambda\left(\mathbb{W}_{\boldsymbol{\beta}}^{d}\right)$ and $\mathbb{Z}^{d}$. Hence, if $\operatorname{wind}_{s}(a)=0$ for all $s=1, \ldots, d$, then $a \in \mathcal{G}_{0}\left(\mathbb{W}_{\boldsymbol{\beta}}^{d}\right)$.

\section{The tRiviality of THE ABStract INDEX GROUP of Banach algebras $\mathcal{W}_{\boldsymbol{\beta}}^{N_{ \pm}}$FOr CERTAin ordered groups $N$}

In this section we prove that for certain ordered groups $N$, the abstract index group of the Banach algebras $\mathcal{A}=\mathcal{W}_{\boldsymbol{\beta}}^{N_{ \pm}}$is trivial, i.e., $\mathcal{G}(\mathcal{A})=\mathcal{G}_{0}(\mathcal{A})$.

Let $N_{1}, \ldots, N_{\mu}$ be additive groups, each of them provided with a linear order $\succeq_{s}$ that makes them into an ordered group. The direct sum

$$
N=N_{1} \oplus \cdots \oplus N_{\mu}
$$

is an additive group. Given $i=\left(i_{1}, \ldots, i_{\mu}\right) \in N$ we define $i \succeq 0$ if and only if $i=0$ or there exists $s=1, \ldots, \mu$ such that

$$
i=\left(i_{1}, \ldots, i_{s}, 0, \ldots, 0\right),
$$

where $i_{1} \in N_{1}, \ldots, i_{s} \in N_{s}$ and $i_{s} \succeq_{s} 0, i_{s} \neq 0$. The relation $\succeq$ is a linear order that makes $N$ into an ordered group. We will call $\succeq$ the anti-lexicographic order on $N$ with respect to the additive ordered groups $N_{1}, \ldots, N_{\mu}$. Our preference for anti-lexicographic order instead of lexicographic order is just for reasons of easier bookkeeping.

Theorem 4.1. Let $N_{1}, \ldots, N_{\mu}$ be additive ordered subgroups of $\mathbb{R}$ with the linear order inherited from $\mathbb{R}$, and let $N$ be the direct sum (4.1) provided with the antilexicographic order. If $\boldsymbol{\beta}$ is an admissible weight on $N$, then $\mathcal{G}\left(\mathcal{W}_{\boldsymbol{\beta}}^{N_{ \pm}}\right)=\mathcal{G}_{0}\left(\mathcal{W}_{\boldsymbol{\beta}}^{N_{ \pm}}\right)$.

Proof. Without loss of generality we carry out the proof only for $\mathcal{W}_{\boldsymbol{\beta}}^{N_{+}}$. The proof goes by induction on $\mu$. For $\mu=0$, there is nothing to prove. Indeed, in this case $N=\{0\}$, and thus $\mathcal{W}_{\boldsymbol{\beta}}^{N_{+}}$is isomorphic to $\mathbb{C}$.

Now let us assume that the assertion has been proved for $\mu-1$, i.e., for

$$
\hat{N}=N_{1} \oplus \cdots \oplus N_{\mu-1} \text {. }
$$

We can identify $\hat{N}$ with the subgroup $\hat{N} \oplus\{0\}$ of $N$. Under this identification $\hat{N}_{+}$ corresponds to the set of all $i \in N_{+}$for which $i_{\mu}=0$. Consequently, the Banach algebra $\mathcal{W}_{\hat{\boldsymbol{\beta}}}^{\hat{N}_{+}}$with $\hat{\beta}_{\left(i_{1}, \ldots, i_{\mu-1}\right)}:=\beta_{\left(i_{1}, \ldots, i_{\mu-1}, 0\right)}$ is isometrically isomorphic to the 
following Banach subalgebra of $\mathcal{W}_{\boldsymbol{\beta}}^{N_{+}}$:

$$
\hat{\mathcal{W}}_{\boldsymbol{\beta}}^{N_{+}}=\left\{\boldsymbol{a} \in \mathcal{W}_{\boldsymbol{\beta}}^{N_{+}}: a_{i}=0 \text { for all } i \in N_{+} \text {with } i_{\mu}>0\right\} .
$$

Hence $\mathcal{G}(\mathcal{A})=\mathcal{G}_{0}(\mathcal{A})$ for $\mathcal{A}=\hat{\mathcal{W}}_{\boldsymbol{\beta}}^{N_{+}}$.

For $r \in[0,1]$ we define the following mapping on $\mathcal{W}_{\boldsymbol{\beta}}^{N_{+}}$:

$$
\phi_{r}:\left\{a_{i}\right\}_{i \in N_{+}} \mapsto\left\{c_{i}\right\}_{i \in N_{+}}, \quad c_{i}= \begin{cases}a_{i} & \text { if } i_{\mu}=0 \\ a_{i} r^{i_{\mu}} & \text { if } i_{\mu}>0 .\end{cases}
$$

In fact, $\phi_{r}$ is a homomorphism on $\mathcal{W}_{\boldsymbol{\beta}}^{N_{+}}$for each $r \in[0,1]$. Moreover, $\phi_{1}(\boldsymbol{a})=\boldsymbol{a}$ and $\phi_{0}(\boldsymbol{a}) \in \hat{\mathcal{W}}_{\boldsymbol{\beta}}^{N_{+}}$. We claim that the mapping $r \in[0,1] \mapsto \phi_{r}(\boldsymbol{a})$ is continuous for each $\boldsymbol{a} \in \mathcal{W}_{\boldsymbol{\beta}}^{N_{+}}$. Indeed, this mapping is continuous for each $\boldsymbol{a}=\boldsymbol{e}_{i}$ with $i \in N_{+}$, the estimate $\left\|\phi_{r}(\boldsymbol{a})\right\| \leq\|\boldsymbol{a}\|$ holds, and the set of finite linear combinations of $\left\{\boldsymbol{e}_{i}\right\}_{i \in N_{+}}$ is dense in $\mathcal{W}_{\boldsymbol{\beta}}^{N_{+}}$.

Since $\phi_{r}$ are homomorphisms, the inverse of $\phi_{r}(\boldsymbol{a})$ is given by $\phi_{r}\left(\boldsymbol{a}^{-1}\right)$, provided $\boldsymbol{a}$ is invertible. Hence $\boldsymbol{a} \in \mathcal{G}\left(\mathcal{W}_{\boldsymbol{\beta}}^{N_{+}}\right)$can be pathwise connected to $\phi_{0}(\boldsymbol{a}) \in$ $\mathcal{G}\left(\hat{\mathcal{W}}_{\boldsymbol{\beta}}^{N_{+}}\right)=\mathcal{G}_{0}\left(\hat{\mathcal{W}}_{\boldsymbol{\beta}}^{N_{+}}\right)$within $\mathcal{G}\left(\mathcal{W}_{\boldsymbol{\beta}}^{N_{+}}\right)$. Consequently, $\boldsymbol{a} \in \mathcal{G}_{0}\left(\mathcal{W}_{\boldsymbol{\beta}}^{N_{+}}\right)$.

The following proposition shows that an ordered group which is group-isomorphic to $\mathbb{Z}^{d}$ is order-preserving group-isomorphic to an ordered group $N$ of the type appearing in Theorem 4.1. We put $\mathbb{R}_{+}=\{p \in \mathbb{R}: p>0\}$.

Proposition 4.2. Let $G$ be an ordered group which is group-isomorphic to $\mathbb{Z}^{d}$. Then there exists an order-preserving group-isomorphism $\phi: G \rightarrow N$, where

$$
N=N_{1} \oplus \cdots \oplus N_{\mu}
$$

is provided with the anti-lexicographic order and $N_{1}, \ldots, N_{\mu}$ are additive subgroups of $\mathbb{R}$, inheriting their order from $\mathbb{R}$. Moreover, there exist $\sigma_{s} \in \mathbb{N}$ and $p_{s} \in \mathbb{R}_{+}^{\sigma_{s}}$ such that

$$
\phi_{s}: \mathbb{Z}^{\sigma_{s}} \rightarrow N_{s}, i_{s} \mapsto\left\langle p_{s}, i_{s}\right\rangle
$$

are group-isomorphisms for each $s=1, \ldots, \mu$.

We remark that $d=\sigma_{1}+\cdots+\sigma_{\mu}$. The numbers $\mu$ and $\sigma_{1}, \ldots, \sigma_{\mu}$ are uniquely determined by the ordered group $G$.

For $s=1, \ldots, \mu$, one can introduce a linear order $\succeq_{s}$ in $\mathbb{Z}^{\sigma_{s}}$ such that $\mathbb{Z}^{\sigma_{s}}$ becomes an ordered group by stipulating that $i_{s} \succeq 0$ for $i_{s} \in \mathbb{Z}^{\sigma_{s}}$ if and only if $\phi_{s}\left(i_{s}\right) \geq 0$. We denote this additive ordered group by $\boldsymbol{Z}_{s}$ and remark that $\phi_{s}: \boldsymbol{Z}_{s} \rightarrow N_{s}$ is an order-preserving group-isomorphism.

Proof. Assume that $G=\mathbb{Z}^{d}$. The proof can be carried out by induction on $d$, where the case $d=0$ is trivial. It is possible to extend the linear order on $\mathbb{Z}^{d}$ to a (not necessarily unique) linear order on $\mathbb{R}^{d}$ that turns $\mathbb{R}^{d}$ into an ordered real vector space [3]. By a result of Erdős [5], there exists a hyperplane $H$, uniquely determined by the order on $\mathbb{Z}^{d}$, such that the corresponding (two) half-spaces contain exclusively positive or negative, resp., elements with respect to the linear order on $\mathbb{R}^{d}$. In other words, there exists a (unique) unit vector $p \in \mathbb{R}^{d}$ such that the following hold: (a) if $i \in \mathbb{Z}^{d}$ and $\langle p, i\rangle>0$, then $i \succ 0$; (b) if $i \in \mathbb{Z}^{d}$ and $\langle p, i\rangle<0$, 
then $i \prec 0$. Denote by $\xi: \mathbb{Z}^{d} \rightarrow \mathbb{R}$ the group-homomorphism $\xi(i)=\langle p, i\rangle$. Obviously, $\operatorname{ker} \xi=H \cap \mathbb{Z}^{d}$ is a subgroup of $\mathbb{Z}^{d}$. The image $N^{\prime}$ of $\xi$ is a finitely generated subgroup of $\mathbb{R}$ without nonzero elements of finite order. Since $N^{\prime}$ can also be represented as the direct sum of finitely many cyclic groups ([10], Corollary to Theorem 4.J), $N^{\prime}$ is group-isomorphic to $\mathbb{Z}^{\sigma}$ for some $\sigma \in\{1, \ldots, d\}$. Choose any generators of $N^{\prime}$, and let $i_{1}, \ldots, i_{\sigma} \in \mathbb{Z}^{d}$ be any pre-images of these generators with respect to $\xi$. Let $K$ be the subgroup of $\mathbb{Z}^{d}$ generated by $i_{1}, \ldots, i_{\sigma}$. Then the restriction of $\xi$ to the ordered group $K$, where the order is inherited from $\mathbb{Z}^{d}$, is an orderpreserving group-isomorphism between $K$ and $N^{\prime}$. Notice that the injectiveness of $\xi$ follows from the absence of nonzero elements of finite order, and the orderpreservation follows from statements (a) and (b) above. From this it follows that $\mathbb{Z}^{d}=\operatorname{ker} \xi \oplus K$. Thus ker $\xi$ is group-isomorphic to $\mathbb{Z}^{d-\sigma}$. The induced isomorphism between $\mathbb{Z}^{d}=\operatorname{ker} \xi \oplus K$ and $\operatorname{ker} \xi \oplus N^{\prime}$ is order-preserving if the order in ker $\xi \oplus N^{\prime}$ is defined in an anti-lexicographic manner. If $\sigma<d$, we apply the same argument once again to $\operatorname{ker} \xi$ instead of $G$. If $\sigma=d$, i.e., $\operatorname{ker} \xi=\{0\}$, we are done.

In order to prove (4.7), recall that $N^{\prime}$ is group-isomorphic to $\mathbb{Z}^{\sigma}$. Any isomorphism is of the kind $i \in \mathbb{Z}^{\sigma} \mapsto\langle q, i\rangle \in \mathbb{R}$, where the entries of $q \in \mathbb{R}^{\sigma}$ are $\mathbb{Q}$-linearly independent. In particular, they are nonzero. A moment's thought reveals that we may assume all entries of $q$ to be positive.

First proof of the implication (a) $\Rightarrow$ (b) of Theorem 2.1. From Proposition 4.2 we obtain that $\mathbb{W}_{\boldsymbol{\beta}}^{d, \pm}$ is isometrically isomorphic to $\mathcal{W}_{\boldsymbol{\gamma}}^{N_{ \pm}}$, where $\boldsymbol{\gamma}_{\phi(i)}=\boldsymbol{\beta}_{i}, i \in \mathbb{Z}^{d}$. Moreover, this implies that we can apply Theorem 4.1. Hence $\mathcal{G}\left(\mathbb{W}_{\boldsymbol{\beta}}^{d, \pm}\right)=\mathcal{G}_{0}\left(\mathbb{W}_{\boldsymbol{\beta}}^{d, \pm}\right)$.

Now assume that condition (a) of Theorem 2.1 is fulfilled. Then $a=a_{-} a_{+}$with $a_{ \pm} \in \mathcal{G}\left(\mathbb{W}_{\boldsymbol{\beta}}^{d, \pm}\right)=\mathcal{G}_{0}\left(\mathbb{W}_{\boldsymbol{\beta}}^{d, \pm}\right)$. Since $\mathcal{G}_{0}(\mathcal{A})=\{\exp (b): b \in \mathcal{A}\}$ for commutative Banach algebras $\mathcal{A}$, the functions $a_{ \pm}$are exponentials of functions in $\mathbb{W}_{\boldsymbol{\beta}}^{d, \pm}$. Thus condition (b) follows.

\section{The Maximal ideal SPACE OF $\mathcal{W}_{\boldsymbol{\beta}}^{N_{ \pm}}$FOR ORDERED Groups $N=\mathbb{Z}^{d}$}

In this section we describe for arbitrary ordered groups $N=\mathbb{Z}^{d}$ and arbitrary admissible weights the maximal ideal space of $\mathcal{W}_{\boldsymbol{\beta}}^{N_{+}}$in terms of the maximal ideal space of $\mathcal{W}_{\boldsymbol{\beta}}^{N}$, or, what is the same (due to Corollary 3.2) and more convenient to us, in terms of the set $K_{\boldsymbol{\beta}}$. A description of $\mathcal{W}_{\boldsymbol{\beta}}^{N_{-}}$can be carried out analogously and will therefore be omitted.

According to Proposition 4.2 and the remarks following it, any ordered group $\mathbb{Z}^{d}$ is order-preserving group-isomorphic to the additive ordered group

$$
N=N_{1} \oplus \cdots \oplus N_{\mu}
$$

with anti-lexicographically defined order, where $N_{s}=\mathbb{Z}^{\sigma_{s}}$ are additive ordered groups with the order $\succeq_{s}$ such that $i_{s} \succeq_{s} 0$ if and only if $\left\langle p_{s}, i_{s}\right\rangle \geq 0$. Here $\mu, \sigma_{1}, \ldots, \sigma_{\mu} \in \mathbb{N}$ and $p_{s}=\left(p_{s, 1}, \ldots, p_{s, \sigma_{s}}\right) \in \mathbb{R}_{+}^{\sigma_{s}}$.

Note that $N$ equals $\mathbb{Z}^{d}$ with $d=\sigma_{1}+\cdots+\sigma_{\mu}$. We will assume without loss of generality that our originally given ordered group $\mathbb{Z}^{d}$ is equal to the ordered group $N$ defined in (5.1). This amounts to considering $\mathbb{Z}^{d}$ provided with a linear order of a special type. However, any arbitrary order on $\mathbb{Z}^{d}$ is equivalent to some linear order of this special type. 
Let $d_{0}=0$ and let $d_{s}=\sigma_{1}+\cdots+\sigma_{s}, s=1, \ldots, \mu$, noting that $d_{\mu}=d$. For $s=1, \ldots, \mu$ we introduce the following subsets of $\mathbb{R}^{d_{s}}$ :

$$
\begin{aligned}
K_{s} & =\left\{\left(x_{1}, \ldots, x_{d_{s}}\right):\left(x_{1}, \ldots, x_{d}\right) \in K_{\boldsymbol{\beta}}\right\}, \\
K_{s, p_{s}} & =\left\{x-\lambda\left(0, \ldots, 0, p_{s, 1}, \ldots, p_{s, \sigma_{s}}\right): x \in K_{s}, \lambda \geq 0\right\} .
\end{aligned}
$$

Moreover, let $\mathcal{C}_{0}=\{0\}$, and for $s=1, \ldots, \mu$, put

$$
\mathcal{C}_{s}=\left\{\left(i_{1}, \ldots, i_{d_{s}}, 0, \ldots, 0\right) \in N_{+}\right\} .
$$

Then $\{0\}=\mathcal{C}_{0} \subset \mathcal{C}_{1} \subset \mathcal{C}_{2} \subset \cdots \subset \mathcal{C}_{\mu}=N_{+}$.

Proposition 5.1. $K_{s}$ is a convex body in $\mathbb{R}^{d_{s}}$, and $K_{s, p_{s}}$ is a closed, convex subset in $\mathbb{R}^{d_{s}}$ containing $K_{s}$. Moreover,

$$
K_{s, p_{s}}=\left\{y_{*} \in \mathbb{R}^{d_{s}}: \sup _{i=\left(i_{*}, 0\right) \in \mathcal{C}_{s}}\left(\left\langle i_{*}, y_{*}\right\rangle-\log \left(\beta_{i}\right)\right)<\infty\right\} .
$$

Proof. The first two statements are trivial. It remains to prove (5.5). Observe that for $x_{*} \in \mathbb{R}^{d_{s}}$

$$
\max _{y_{*} \in K_{s}}\left\langle x_{*}, y_{*}\right\rangle=\max _{y \in K_{\boldsymbol{\beta}}}\left\langle\left(x_{*}, 0\right), y\right\rangle=F\left(\left(x_{*}, 0\right)\right),
$$

where $F$ is the supporting function of $K_{\boldsymbol{\beta}}$. Define

$$
\hat{F}_{s}\left(x_{1}, \ldots, x_{d_{s}}\right)= \begin{cases}F\left(x_{1}, \ldots, x_{d_{s}}, 0, \ldots, 0\right) & \text { if } \phi_{s}\left(x_{d_{s-1}+1}, \ldots, x_{d_{s}}\right) \geq 0 \\ +\infty & \text { if } \phi_{s}\left(x_{d_{s-1}+1}, \ldots, x_{d_{s}}\right)<0\end{cases}
$$

where $\phi_{s}$ is given by (4.7). We obtain that for $x_{*}=\left(x_{1}, \ldots, x_{s}\right) \in \mathbb{R}^{\sigma_{1}} \oplus \cdots \oplus \mathbb{R}^{\sigma_{s}}=$ $\mathbb{R}^{d_{s}}$

$$
\max _{y_{*} \in K_{s, p_{s}}}\left\langle x_{*}, y_{*}\right\rangle=\max _{y_{*} \in K_{s}, \lambda \geq 0}\left(\left\langle x_{*}, y_{*}\right\rangle-\lambda\left\langle x_{s}, p_{s}\right\rangle\right)=\hat{F}_{s}\left(x_{*}\right) .
$$

Hence $\hat{F}_{s}$ is the supporting function of the closed, convex set $K_{s, p_{s}}$. Therefore [12],

$$
K_{s, p_{s}}=\left\{y_{*} \in \mathbb{R}^{d_{s}}:\left\langle x_{*}, y_{*}\right\rangle \leq \hat{F}_{s}\left(x_{*}\right) \text { for all } x_{*} \in \mathbb{R}^{d_{s}}\right\} .
$$

Assume $y_{*} \in K_{s, p_{s}}$. Then $\left\langle x_{*}, y_{*}\right\rangle \leq F\left(\left(x_{*}, 0\right)\right)$ for all $x_{*}=\left(x_{1}, \ldots, x_{s}\right) \in \mathbb{R}^{d_{s}}$ with $\phi_{s}\left(x_{s}\right) \geq 0$. If $i=\left(i_{*}, 0\right) \in \mathcal{C}_{s}$, then this applies to $x_{*}=i_{*}$. Since $F(i) \leq \log \left(\beta_{i}\right)$, it follows that $y_{*}$ is contained in the set defined by the right-hand side of 5.5 .

Conversely, let $y_{*}$ belong to the set given by the right-hand side of (5.5). Then $\left\langle i_{*}, y_{*}\right\rangle \leq \log \left(\beta_{i}\right)+M$ for all $i=\left(i_{*}, 0\right) \in \mathcal{C}_{s}$ and some constant $M$. Replace $i$ by $n i$, divide by $n$ and take the limit as $n \rightarrow \infty$. We obtain that $\left\langle i_{*}, y_{*}\right\rangle \leq F(i)$ for all $i=\left(i_{*}, 0\right) \in \mathcal{C}_{s}$. In particular, this holds for all $i=\left(i_{1}, \ldots, i_{s}, 0, \ldots, 0\right) \in \mathbb{Z}^{d}$ with $\phi_{s}\left(i_{s}\right)>0$. Thus $\left\langle x_{*}, y_{*}\right\rangle \leq F(x)$ for all $x=\left(x_{*}, 0\right)=\left(x_{1}, \ldots, x_{s}, 0, \ldots, 0\right) \in \mathbb{Q}^{d}$ with $\phi_{s}\left(x_{s}\right)>0$. A continuity argument finally shows that $y_{*} \in K_{s, p_{s}}$.

Now we introduce for $s=1, \ldots, \mu$ the following subsets of $\mathbb{C}^{d}$ :

$$
\Omega_{\boldsymbol{\beta}, s}=\left\{\left(t_{1} e^{x_{1}}, \ldots, t_{d_{s}} e^{x_{d_{s}}}, 0, \ldots, 0\right): t_{k} \in \mathbb{T},\left(x_{1}, \ldots, x_{d_{s}}\right) \in K_{s}\right\},
$$

$\Omega_{\boldsymbol{\beta}, 0}=\{0\}$, and

$$
\Omega_{\boldsymbol{\beta}, s}^{+}=\left\{\left(t_{1} e^{x_{1}}, \ldots, t_{d_{s}} e^{x_{d_{s}}}, 0, \ldots, 0\right): t_{k} \in \mathbb{T},\left(x_{1}, \ldots, x_{d_{s}}\right) \in K_{s, p_{s}}\right\} .
$$


It is easy to see that $\Omega_{\boldsymbol{\beta}, s} \subset \Omega_{\boldsymbol{\beta}, s}^{+}$and $\operatorname{clos} \Omega_{\boldsymbol{\beta}, s}^{+}=\Omega_{\boldsymbol{\beta}, s}^{+} \cup \Omega_{\boldsymbol{\beta}, s-1}$ for all $s=1, \ldots, \mu$. The sets $\Omega_{\boldsymbol{\beta}, s}$ are closed, and $\Omega_{\boldsymbol{\beta}, 0}, \Omega_{\boldsymbol{\beta}, 1}^{+}, \ldots, \Omega_{\boldsymbol{\beta}, \mu}^{+}$are mutually disjoint. Put

$$
\Omega_{\boldsymbol{\beta}}^{+}=\Omega_{\boldsymbol{\beta}, 0} \cup \Omega_{\boldsymbol{\beta}, 1}^{+} \cup \cdots \cup \Omega_{\boldsymbol{\beta}, \mu}^{+} .
$$

Theorem 5.2. The maximal ideal space of $\mathcal{W}_{\boldsymbol{\beta}}^{N_{+}}$is homeomorphic to the set $\Omega_{\boldsymbol{\beta}}^{+}$ endowed with the topology induced by $\mathbb{C}^{d}$. In fact, for $z \in \Omega_{\boldsymbol{\beta}, s}^{+}$the corresponding multiplicative linear functional $T_{z}$ is defined by

$$
T_{z}: \boldsymbol{a}=\left\{a_{i}\right\}_{i \in n} \mapsto \sum_{i=\left(i_{1}, \ldots, i_{d_{s}}, 0, \ldots, 0\right) \in \mathcal{C}_{s}} a_{i} z_{1}^{i_{1}} \cdots z_{d_{s}}^{i_{d_{s}}}
$$

and by $T_{0}: \boldsymbol{a} \mapsto a_{0}$ for $z=0 \in \Omega_{\boldsymbol{\beta}, 0}$.

Proof. In order to prove that the maximal ideal space of $\mathcal{W}_{\boldsymbol{\beta}}^{N_{+}}$is equal to $\Omega_{\boldsymbol{\beta}}^{+}$, let us first remark that there is a one-to-one correspondence between the maximal ideals of $\mathcal{W}_{\boldsymbol{\beta}}^{N_{+}}$and the sequences $\boldsymbol{h}=\left\{h_{i}\right\}_{i \in N_{+}}$of complex numbers $h_{i}$ satisfying $h_{i+j}=h_{i} h_{j}$ $\left(i, j \in N_{+}\right), h_{0}=1$ and $\sup _{i \in N_{+}}\left(\left|h_{i}\right| / \beta_{i}\right)<\infty$. In fact, to each such $\boldsymbol{h}$, there corresponds the multiplicative linear functional $T_{\boldsymbol{h}}$ given by $T_{\boldsymbol{h}}: \boldsymbol{a} \mapsto \sum_{i \in N_{+}} a_{i} h_{i}$.

Now let $\boldsymbol{h}$ be such a sequence. Assume that $h_{i}=0$ for a certain $i \in \mathcal{C}_{s+1} \backslash \mathcal{C}_{s}$. Then for each $j \in N_{+} \backslash \mathcal{C}_{s}$, there exists $n \in \mathbb{N}$ such that $n j \succ i$. Thus $h_{j}^{n}=$ $h_{n j-i} h_{i}=0$ and hence $h_{j}=0$. Choosing a particular $i$ as above in such a way that $s$ is as small as possible, we can conclude that for each $\boldsymbol{h}$ there exists a unique $s \in\{0, \ldots, \mu\}$ such that $h_{i} \neq 0$ for all $i \in \mathcal{C}_{s}$ and $h_{i}=0$ for all $i \in N_{+} \backslash \mathcal{C}_{s}$.

Each $i \in \mathcal{C}_{s}$ can be represented in the form $i=\sum_{t=1}^{d_{s}} i_{t} e_{t}$. Putting $z_{t}:=h_{e_{t}} \neq 0$, it follows that $h_{i}=z_{1}^{i_{1}} \cdots z_{d_{s}}^{i_{d_{s}}}$. Here we use that $h_{i} \neq 0$ for $i \in \mathcal{C}_{s}$. Moreover,

$$
\sup _{i=\left(i_{1}, \ldots, i_{d_{s}}, 0, \ldots, 0\right) \in \mathcal{C}_{s}} \frac{\left|z_{1}\right|^{i_{1}} \cdots\left|z_{d_{s}}\right|^{i_{d_{s}}}}{\beta_{i}}<\infty .
$$

From this we see that there is indeed a one-to-one correspondence between the maximal ideal space and the set $\Omega_{\boldsymbol{\beta}}^{+}$and that the multiplicative linear functionals specifying the maximal ideals are given by (5.13).

In order to prove that the natural topology of the maximal ideal space coincides with the topology of $\Omega_{\boldsymbol{\beta}}^{+}$induced by $\mathbb{C}^{d}$, we rely on the result used in the proof of Theorem 3.4. First note that the sets $\Omega_{\boldsymbol{\beta}, s}^{+} \cup \Omega_{\boldsymbol{\beta}, s-1}$ are compact subsets of $\mathbb{C}^{d}$, and

$$
\Omega_{\boldsymbol{\beta}}^{+}=\bigcup_{s=1}^{\mu}\left(\Omega_{\boldsymbol{\beta}, s-1} \cup \Omega_{\boldsymbol{\beta}, s}^{+}\right)
$$

Hence $\Omega_{\boldsymbol{\beta}}^{+}$is compact, too.

It remains to prove that the mapping $z \in \Omega_{\boldsymbol{\beta}}^{+} \mapsto T_{z}(\boldsymbol{a}) \in \mathbb{C}$ is continuous for each fixed $\boldsymbol{a} \in \mathcal{W}_{\boldsymbol{\beta}}^{N_{+}}$. Since $\sup _{z \in \Omega_{\boldsymbol{\beta}}^{+}}\left|T_{z}(\boldsymbol{a})\right| \leq\|\boldsymbol{a}\|_{\mathcal{W}_{\boldsymbol{\beta}}^{N_{+}}}$, it suffices to prove the continuity only for the elements $\boldsymbol{a}=\boldsymbol{e}_{i}, i \in N_{+}$, whose linear span is dense in $\mathcal{W}_{\boldsymbol{\beta}}^{N_{+}}$. Moreover, since, by (5.15), $\Omega_{\boldsymbol{\beta}}^{+}$is the finite union of compact subsets of $\mathbb{C}^{d}$, it is enough to prove the continuity of the restrictions of the functions $z \mapsto T_{z}\left(\boldsymbol{e}_{i}\right)$ to each of the sets $\Omega_{\boldsymbol{\beta}, s-1} \cup \Omega_{\boldsymbol{\beta}, s}^{+}$for each $s=1, \ldots, \mu$ and each $i \in N_{+}$. 
Let us first recall that

$$
T_{z}\left(\boldsymbol{e}_{i}\right)= \begin{cases}z_{1}^{i_{1}} \cdots z_{d_{s}}^{i_{s}} & \text { if } z \in \Omega_{\boldsymbol{\beta}, s}^{+} \text {and } i \in \mathcal{C}_{s}, \\ z_{1}^{i_{1}} \cdots z_{d_{s-1}}^{i_{s-1}} & \text { if } z \in \Omega_{\boldsymbol{\beta}, s-1} \text { and } i \in \mathcal{C}_{s-1}, \\ 0 & \text { if }\left(z \in \Omega_{\boldsymbol{\beta}, s}^{+} \text {and } i \notin \mathcal{C}_{s}\right) \text { or }\left(z \in \Omega_{\boldsymbol{\beta}, s-1} \text { and } i \notin \mathcal{C}_{s-1}\right) .\end{cases}
$$

The continuity is trivial apart from the case where $i \in \mathcal{C}_{s} \backslash \mathcal{C}_{s-1}$. In this case, we have to prove that $T_{z}\left(\boldsymbol{e}_{i}\right) \rightarrow 0$ if $z \in \Omega_{\boldsymbol{\beta}, s}^{+}$tends to a point in $\Omega_{\boldsymbol{\beta}, s-1}$.

Indeed, let us write $z=\left(z_{1}, \ldots, z_{d_{s}}, 0, \ldots, 0\right) \in \Omega_{\boldsymbol{\beta}, s}^{+}$in the form $z_{k}=t_{k} \exp \left(x_{k}\right)$, $1 \leq k \leq d_{s-1}$, and $z_{d_{s-1}+k}=t_{d_{s-1}+k} \exp \left(x_{d_{s-1}+k}-\lambda p_{s, k}\right), 1 \leq k \leq \sigma_{s}$. Note that $p_{s, k}>0$. Since $x_{*}=\left(x_{1}, \ldots, x_{d_{s}}\right)$ is taken from the bounded set $K_{s}, z$ converges to a point in $\Omega_{\boldsymbol{\beta}, s-1}$ if and only if $\lambda \rightarrow+\infty$. Now

$$
\log \left|T_{z}\left(\boldsymbol{e}_{i}\right)\right|=\left\langle x_{*}, i_{*}\right\rangle-\lambda\left\langle p_{s}, i_{s}\right\rangle,
$$

where $i=\left(i_{*}, 0\right)=\left(i_{1}, \ldots, i_{s}, 0, \ldots, 0\right)$, tends to $-\infty$ since $\phi_{s}\left(i_{s}\right)=\left\langle p_{s}, i_{s}\right\rangle>0$.

Corollary 5.3. The compact space $\Omega_{\boldsymbol{\beta}}^{+}$is contractible to the point $0 \in \Omega_{\boldsymbol{\beta}, 0}$.

Proof. Let $\hat{\Omega}_{\boldsymbol{\beta}, 0}^{+}=\{0\}$ and $\hat{\Omega}_{\boldsymbol{\beta}, s}^{+}=\Omega_{\boldsymbol{\beta}, 0} \cup \Omega_{\boldsymbol{\beta}, 1}^{+} \cup \cdots \cup \Omega_{\boldsymbol{\beta}, s}^{+}$. Since $\hat{\Omega}_{\boldsymbol{\beta}, \mu}^{+}=\Omega_{\boldsymbol{\beta}}^{+}$, it suffices to prove that all the spaces $\hat{\Omega}_{\boldsymbol{\beta}, s}^{+}$are homotopically equivalent.

In fact, we can even be more specific. For each $s=1, \ldots, \mu, \hat{\Omega}_{\beta, s-1}^{+}$is a strong deformation retract of $\hat{\Omega}_{\beta, s}$ (cf. [13]). The retracting deformation is given by

$$
F_{r}:\left(z_{1}, \ldots, z_{s-1}, z_{s}, 0, \ldots, 0\right) \in \hat{\Omega}_{\boldsymbol{\beta}, s}^{+} \mapsto\left(z_{1}, \ldots, z_{s-1}, z_{s}(r), 0, \ldots, 0\right) \in \hat{\Omega}_{\boldsymbol{\beta}, s}^{+},
$$

where $\left[z_{s}(r)\right]_{k}:=\left[z_{s}\right]_{k}(1-r)^{p_{s, k}}\left(k=1, \ldots, \sigma_{s} ; r \in[0,1]\right)$. Notice that $p_{s, k}>0$.

Second proof of the implication (a) $\Rightarrow$ (b) of Theorem 2.1. By Corollary 5.3 the cohomotopy group $\pi^{1}\left(\Omega_{\boldsymbol{\beta}}^{+}\right)$is trivial. Hence from Theorem 5.2 and Theorem 3.5 it follows that $\mathcal{G}(\mathcal{A})=\mathcal{G}_{0}(\mathcal{A})$ for $\mathcal{A}=\mathcal{W}_{\boldsymbol{\beta}}^{N_{+}}$where $N=\mathbb{Z}^{d}$ is an ordered group. The corresponding statement holds also for $\mathcal{A}=\mathcal{W}_{\boldsymbol{\beta}}^{N_{-}}$. Now the proof can be completed in the same way as the first proof of this implication given in Section 4

\section{REFERENCES}

1. R. Arens, The group of invertible elements of a commutative Banach algebra, Studia Math. Zeszyt (Ser. Specjalna) 1 (1963), 21-23. MR 26:4198

2. A. Böttcher and B. Silbermann, Analysis of Toeplitz Operators, Springer, Berlin, 1990. MR 92e: 47001

3. L. Cerlienco and M. Mureddu, Rappresentazione matriciale degli ordini l.c. su $\mathbb{R}^{n}$ e su $\mathbb{N}^{n}$, Rend. Sem. Fac. Sc. Univ. Cagliari 66 (1996), 49-68. MR 98c:06025

4. R.G. Douglas, Banach Algebra Techniques in Operator Theory, Graduate Texts in Mathematics, vol. 179, Springer, Berlin, 1998. MR 99c:47001

5. J. Erdős, On the structure of ordered real vector spaces, Publ. Math. Debrecen 4 (1956), 334-343. MR 18:137b

6. I. Gelfand, D. Raikov, and G. Shilov, Commutative Normed Rings, AMS Chelsea Publ., Providence, RI, 1964. MR 34:4940

7. I. Gohberg, S. Goldberg, and M.A. Kaashoek, Classes of Linear Operators, Vol. II, Birkhäuser, vol. OT 63, Basel-Boston, 1993. MR 95a:47001

8. T.N.T. Goodman, C.A. Micchelli, G. Rodriguez, and S. Seatzu, On the Cholesky factorization of the Gram matrix of multivariate functions, SIAM J. Matrix Anal. Appl. 22 (2000), 501-526. MR 2001k:15020

9. H. Helson and D. Lowdenslager, Prediction theory and Fourier series in several variables, Acta Math. 99 (1958), 165-202; part II: Acta Math. 106 (1961), 175-213. MR 20:4155 MR 31:562 
10. I. N. Herstein, Topics in Algebra, Xerox College Publ., Waltham, Mass., 1964. MR 50:9456

11. E. Hille and R.S. Phillips, Functional Analysis and Semigroups, Amer. Math. Soc. Coll. Publ., vol. 31, Amer. Math. Soc., Providence, RI, 1957. MR 19:664d

12. J.-B. Hiriart-Urruty and C. Lemaréchal, Convex Analysis and Minimization Algorithms I, Springer, Berlin, 1993. MR 95m:90001

13. Sze-tsen Hu, Homotopy Theory, Pure and Applied Mathematics, vol. 8, New York, Academic Press, 1959. MR 21:5186

14. M.G. Krein, Integral equations on the half-line with a kernel depending on the difference of the arguments, AMS Transl. Ser. 2, 22 (1962), 163-288; transl. from Uspehi Mat. Nauk. 13 (1958), 3-120. MR 21:1507

15. C.V.M. van der Mee, G. Rodriguez, and S. Seatzu, Spectral factorization of bi-infinite multiindex block Toeplitz matrices, Linear Algebra and its Applications 343-344 (2002), 355-380.

16. M.A. Naŭmark, Normed algebras, Wolters-Noordhoff Publ., Groningen, 1972. MR 55:11042

17. H.L. Royden, Function algebras, Bull. Amer. Math. Soc. 69 (1963), 281-298. MR 26:6817

18. W. Rudin, Fourier Analysis on Groups, John Wiley, New York, 1962. MR 27:2808

19. I.B. Simonenko, On multidimensional discrete convolutions, Matem. Issled. Kishinev 3(1) (1968), 108-122 [Russian]. MR 41:2412

20. J.L. Taylor, Banach algebras and topology, In: Algebras in Analysis, Academic Press, London 1975, pp. 118-186. MR 54:5837

Fakultät für Mathematik, Technische Universität Chemnitz, 09107 Chemnitz, GerMANY

E-mail address: tehrhard@mathematik.tu-chemnitz.de

Dipartimento di Matematica, Università di Cagliari, via Ospedale 72, 09124 Cagliari, ITALY

E-mail address: cornelis@bugs.unica.it 